\title{
Energy budget of an implantable glucose measurement system
}

\author{
Thomas Basmer, Dieter Genschow, Marlen Froehlich*, Mario Birkholz \\ IHP, Frankfurt (Oder), Germany, basmer@ihp-microelectronics.com
}

\begin{abstract}
The theoretical evaluation of an implantable system measuring glucose for diabetes diagnostics and therapy has recently been presented. This work reports on the realisation of the proposed system on a printed circuit board and measurements of its energy budget. It is shown that the system can be run with small $3.2 \mathrm{~V}$ batteries for more than six months with an average energy consumption of $0.12 \mathrm{mAh}$.
\end{abstract}

\section{Introduction}

Implantable medical systems widely in use, like pacemakers, defibrillators and insulin pumps have paved the way to ever more complex applications of implants, e.g. artificial retina, intelligent hip prosthesis etc. $[1,2]$. Recently the architecture of an implantable sensor system measuring glucose concentration and temperature in the subcutaneous tissue has been outlined [3]. Here we present the measured power consumption of the system, which is one of the decisive parameters of implantable devices governing their operational lifetime. In the first part of this work, the hardware components of the glucose monitor will be detailed, while section 2 will describe the application and operation scenario. Measurement methods for the energy budget and results will be presented in section 3 and 4, respectively. At the end the conclusions and an outlook are given.

\subsection{The glucose measurement system}

As can be seen in Figure 1 the monitor system consists of four main parts: sensor, microcontroller, radio module with antenna and a battery for power supply, which were all installed on or connected to a printed circuit board (PCB). The following subsections will describe the individual components in more detail.

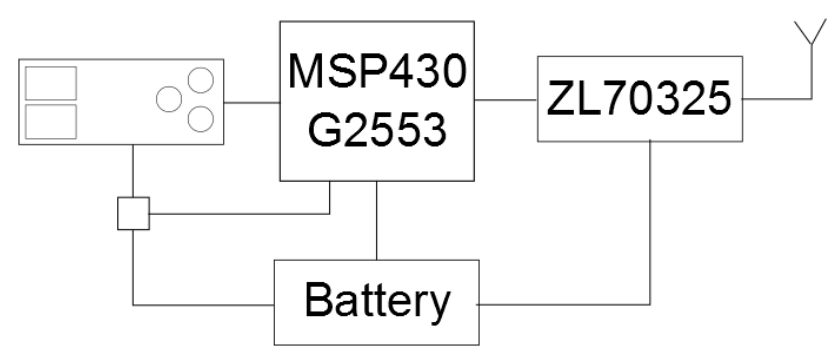

Figure 1 Block diagram of the implantable glucose measurement system encompassing the sensor chip, microcontroller, radio module and power supply

\subsubsection{Sensor chip}

The sensor is based on a micro-electro-mechanical system (MEMS) using a biochemical assay of dextran and concanavalin $\mathrm{A}$, the viscosity of which depend on glucose con- centration [4]. A cantilever is moved through this sensoric liquid and the time is measured it takes until the cantilever reaches a defined position. The measured switching time can be transformed into glucose concentration. The typical switching time for normal glucose concentration lies between 50 and $200 \mathrm{~ms}$. The switching time can be adjusted by changing an external control voltage $\left(V_{c t r l}\right)$. The sensor chip consumes about $30 \mathrm{~mA}$. A glucose measurement cycle is started by, first activating $V_{c t r l}$, which is followed by supplying a working voltage $V_{d d}$ of $3.3 \mathrm{~V}$. The measurement is finished when the cantilever has reached its final position and the sensor chip switches off $V_{d d}$. The second functionality is the measurement of the tissue temperature. For this purpose $V_{d d}$ must be activated and the flowing current $(130-190 \mu \mathrm{A})$ representing the temperature $(0$ $100{ }^{\circ} \mathrm{C}$ ) must be measured. The sensor chip consumes no energy when not in the active state.

\subsubsection{Microcontroller}

The microcontroller is an MSP430G2553 from TI. It is a low-power system (active $330 \mu \mathrm{A}$, standby $0.5 \mu \mathrm{A}$, off mode $0.1 \mu \mathrm{A}$ ) and is well suited for this application [5]. It encompasses with on-chip comparators, analog digital converters (ADCs), two 16-bit timers and $16 \mathrm{kB}$ flash memory. The microcontroller is used to control the sensor chip, measure the switching time and temperature and communicate with the radio module. The switching time is measured using an internal timer and the interrupt capability of a general purpose input output (GPIO) pin of the microcontroller. The timer is started after turning $V_{c t r l}$ and $V_{d d}$ on. When the measurement has finished the sensor chip switches off $V_{d d}$ that stops the current flow through the sensor chip. This current drop is transformed into a voltage drop using a comparator to trigger an interrupt at a GPIO to stop the timer. For a precise temperature measurement it is necessary to measure the current changes with $10 \mathrm{nA}$ precision. Therefore, a special temperature measurement method was developed. To reduce the power consumption of the system and to expand the lifetime the microcontroller low-power modes should be used whenever possible. The microcontroller and radio module are both clocked by a $24 \mathrm{MHz}$ crystal oscillator. 


\subsubsection{Radio chip}

The transceiver chip ZL70325 from microsemi (previously Zarlink) has been selected for the system presented here, which operates as a Medical Implant Communication Standard (MICS) radio [6]. It has particularly been developed for use in implantable systems sending and receiving in the $402-405 \mathrm{MHz}$ band. For long system life time it comes with low power consumption (Transmit/Receive $5 \mathrm{~mA}$, idle $1 \mathrm{~mA}$, sleep $10 \mathrm{nA}$ ) and a wake up system. When the radio is in sleep mode a $2.4 \mathrm{GHz}$ extreme low power radio $(290 \mathrm{nA})$ searches in an adjustable period for wake up messages on the carrier. If such a message is received it turns on the full radio. The radio is feed using a $24 \mathrm{MHz}$ crystal oscillator and is connected to the microcontroller via a serial peripheral interface (SPI). All radio modules can be setup with a company identification number (ID) and a device ID via software. It is thus possible to clearly identify and address each module separately. The radio also disposes of an extensive register set to setup and calibrate the radio module to be adaptable for various applications.

The packet structure of a typical MICS band transmission is taken from [4]. A data packet has a minimum length of 333 bit ( $\sim 2$ byte) containing up to 14 bytes of data. The package structure starts with a training sequence of at least 8 bits followed by a synchronization part (40 bits), the header containing transceiver ID, flow control, etc. (76 bits), cyclic redundancy check (CRC) checksum (24 bits), 30 bit Reed Solomon code to detect and repair bit errors in the package and up to 31 data blocks (113 data bits, 12 bit CRC, 30 bits Reed Solomon).

\subsubsection{Battery}

As power supply a Litronik pacemaker battery is used [7]. The battery case is hermetically sealed and of $31 \times 28 \times 5 \mathrm{~mm}$ size. It provides $3.2 \mathrm{~V}$ and has a nominal capacity of $1200 \mathrm{mAh}$, but the Litronik data sheet specifies an output voltage above $3 \mathrm{~V}$ for a reduced capacity of 500$600 \mathrm{mAh}$ [7].

\section{Application}

The addressed application scenario is to implant the system and measure glucose and the temperature of the surrounding tissue every 5 minutes. For laboratory tests and higher measurement frequency the interval can be lowered to $10 \mathrm{~s}$. The implant has a communication link to a base station to exchange data and control information. In order to save energy all components of the implantable system are switched off or brought to low-power modes if not used. Two methods are available to switch on the system for measurements as described in the subsections below.

\subsection{Measurement control via base station}

In the first method the sensor is switched off; the radio module is in sleep mode $(10 \mathrm{nA})$ and the microcontroller is in the deepest low power mode (LPM4), where no clock is running and all internal components are switched off. The system is woken up for measurements using the wake up radio and the base station module. The base station sends a $2.4 \mathrm{GHz}$ strobe, containing implant and company ID, to wake up the specified implant. The addressed radio starts up and sends a $403 \mathrm{MHz}$ wake up response. When the radio is ready it generates an interrupt waking up the microcontroller. Subsequently, either this control information can be exchanged or a measurement starts. It is possible to perform a separate temperature and glucose measurement, but it is recommended to do both in common. The tissue temperature is important, since it affects the viscosity of the liquid. After that the measured data is transmitted to the base station.

\subsection{Internal Measurement control}

The second method is used if there is no base station available all the time. Between two measurements the sensor is switched off, the radio module is in sleep mode and the microcontroller in a higher low power mode (LPM3) one low frequency clock is running at $12 \mathrm{kHz}$ and can be used to feed the timer. It is necessary to control the period between two measurements. If the timing interval has passed the microcontroller goes to its active mode and starts the measurements as described above. After that the measured data is stored in the memory of the microcontroller. If the implant is near a base station module its radio is woken up using a wake up strobe of the base station and control information and measurement data can be exchanged. This measurement method is more energy consuming, because the microcontroller cannot go to its deepest low-power mode. But the difference between both methods is very small and not measureable because of the PCBs leakage current (see section 4).

\subsection{Measurement scenario}

Our typical glucose measurement scenario has a measurement interval of five minutes. The measurement cycle starts with a temperature measurement of the tissue. This measurement lasts for $20 \mathrm{~ms}$ and is necessary for a correct transformation of the measured switching time into glucose concentration. The glucose measurement starts immediately after the temperature measurement. The runtime depends on the glucose concentration and is typically between 50 and $200 \mathrm{~ms}$. The measurement data is stored inside the memory of the microcontroller or transmitted directly to the base station. The transmitted data contains the implants ID and all measurement data. Following the packet structure of the radio module, presented 1.1.3 the length of the packet is 333 bit ( $\sim 2$ byte) using the scenario in 2.1. If the scenario described in 2.2 is used the amount of the packets depends on the amount of measured data stored in the internal memory. If we assume that the base station gets access to the implant once a day the amount of data to send is two measurements, each of seven byte length, per data block. Each packet contains up to 31 data blocks. Doing 288 measurements a day a data volume 
of 33 packets (1374 bytes) must be transmitted at once. Until now the data of one measurement is seven bytes containing one status byte, two bytes pre-measured temperature, two bytes switching time and two byte post-measured temperature. The pre-measured temperature is taken as the temperature of the surrounding without the influence of the sensors heating. The switching time is the glucose dependent measuring time of the sensor. The post-measured temperature can be taken after the glucose measurement to get the heating of the surrounding influenced by the sensor. The time between the glucose measurement and the temperature measurement is adjustable to trace the cooling process of the sensor. This is interesting for test purposes only and can be skipped in the real application. The status byte contains the current status of the implant.

\section{$3 \quad$ Methods}

Energy measurements were done using a System SourceMeter® 2636 from Keithley. It was used as power supply and to measure the current consumption with nA precision. Energy consumptions were separately measured for the different system modes and the results are given in Table 1.

Table 1 Operating modes of the implant with the operating states of the different components

\begin{tabular}{|l|c|c|c|}
\hline \multicolumn{1}{|c|}{ Mode } & Radio & Microcontroller & Sensor \\
\hline Sleep 1 & off & LPM4 & off \\
\hline Sleep 2 & off & LPM3 & off \\
\hline TX/RX & on & On & off \\
\hline Temperature & off & On & on \\
\hline Glucose & off & On & on \\
\hline
\end{tabular}

The Sleep mode 1 is used in the application scenario described in 2.1 and Sleep mode 2 is used in the scenario presented in 2.2. The transmit/receive (TX/RX) mode encompasses the energy consumption of a whole communication containing wake-up, receiving control data and sending one data packet containing the measurement data (333 bits). In the Temperature mode the microcontroller uses the temperature circuit of the sensor chip and its internal timers to measure the temperature of the surrounding. The sensors switching time is measured in the Glucose mode.

The power consumption is calculated by multiplying the measured current with the supply voltage of the PCB of $3.3 \mathrm{~V}$. The energy budget is the sum of the power consumptions of all modes and the conversion in percentage for all states. The portion on the lifetime of the different states is calculated using the timings of the application scenario described in 2.1 .

\section{$4 \quad$ Results}

The power consumption of the different system modes are summarized in table 2 .

Table 2 Operating modes of the implant with their power consumption

\begin{tabular}{|l|c|}
\hline \multicolumn{1}{|c|}{ Mode } & Power consumption in $\mathbf{~ m W}$ \\
\hline Sleep 1 & 0.23 \\
\hline Sleep 2 & 0.23 \\
\hline TX/RX & 18.15 \\
\hline Temperature & 0.85 \\
\hline Glucose & 99.0 \\
\hline
\end{tabular}

The power consumption of the two sleep modes may be regarded as wasted energy because the system does nothing than waiting. But looking at the application scenario the system is most of its lifetime in the sleep state accounting for $0,19 \%$ of the systems energy budget. The Sleep mode 1 has the lowest power consumption of $0.23 \mathrm{~mW}$. Sleep mode 2 has also a power consumption of $0.23 \mathrm{~mW}$. The microcontroller is running with low frequency clock of about $12 \mathrm{kHz}$ and feeds the timer with $1.5 \mathrm{kHz}$ but the leakage current of the remaining board components is higher than the difference between LPM3 and LPM4 of the MSP430 microcontroller. So a difference could not be really measured. In TX/RX mode a communication link is established using the wake-up circuit and a data packet of 333 bit length containing control data is send from the base station to the implant and measured data vice versa. The power consumption of this mode is $18.15 \mathrm{~mW}$ and corresponds to $15.65 \%$ of the systems energy budget using the scenario of 2.1. During a $20 \mathrm{~ms}$ temperature measurement the microcontroller and the temperature measurement circuit are active consuming $0.85 \mathrm{~mW}$ of energy representing $0.72 \%$ of the energy budget. The glucose measurement mode is the most energy consuming one. During measurement the microcontroller and the sensor chip are both active, which consumes $99 \mathrm{~mW}$. That is $83.73 \%$ of the energy budget when the sensor chip is active for approximately $200 \mathrm{~ms}$.

Assuming the scenario described above, it can be summarized that the implant is in one of the sleep modes for $99.925 \%$ of its lifetime, while it performs for $0.067 \%$ and $0.0067 \%$ a glucose or temperature measurement, respectively, and demands $0.0012 \%$ for the TX/RX mode.

The measurements and calculations show that the presented system can be run for six month using the battery presented in 1.1.4 and one of the both scenarios presented in 2.1 and 2.2. In such scenarios requiring long system lifetimes not only the energy consumption is important to look at also the portion on the energy budget is very important. The sleep modes are the modes with the lowest energy consumption but with the highest part on the on system lifetime of about $99 \%$. To significantly optimize the system lifetime the sleep modes power consumption must be 
reduced to have a significant impact on the systems lifetime. The second state to optimize is the glucose measurement state. Therefore, the sensor must be optimized by reducing measurement time or the sensors power consumption of up to $1 \mathrm{~mW}$.

\section{Conclusion}

This paper presented the measurement of the power consumption of an implantable glucose measurement system build up on a PCB. The system measures the glucose concentration using a fully embedded BioMEMS controlled by a MSP430 microcontroller from Texas Instruments. For wireless data transmission a MICS band radio from microsemi is used sending and receiving in the $403 \mathrm{MHz}$ band released for the use by implantable systems. It has been shown that it is possible to build up such a system with an average energy consumption of $0.12 \mathrm{mAh}$ for a realistic scenario. It is possible to run the system for more than six months by using an implantable battery developed for pacemakers.

It could be shown that in the applied target scenario the sleep mode, where the system does nothing but waiting for the next measurement and consuming $0.23 \mathrm{~mW}$ of power with a part of $0.19 \%$ of the systems energy budget but $99 \%$ of the system lifetime, is the most wasting part. The sleep mode has to be optimized in order to realize a significant increase of the system lifetime. In addition, the glucose measurement state with a power consumption of $99 \mathrm{~mW}$ and $83.73 \%$ of the full energy budget requires optimization. This might be achieved by reducing either the glucose measurement cycle or the sensors current consumption of $30 \mathrm{~mA}$.

In future work the PCB must be shrunk to a size of a typical pacemaker size and below $(30 \times 40 \mathrm{~mm})$. The other task is to reduce the energy consumption of the sleep modes taking care on the used components on the PCB like pull-up resistors and conductors. In general, this first draft of an implantable glucose monitor system still offers space for improvement, i.e. reducing the form factor and extending the life time.

\section{Acknowledgements}

The investigations presented here were performed within the development project of an implantable glucose sensor funded by the BMBF under contract number 16SV3934 (Voll-implantierbarer Glucosesensor für Diabetesdiagnostik und -therapie - GlucoPlant).

\section{$7 \quad$ References}

[1] A. Harscher, U. Greppmaier, W.-G. Wrobel, K. Stingl, E. Zrenner, Das drahtlose Retina Implantat im klinischen Test, In:, Energieautarke Sensorik 2010 - Mikrosensorik mit autarker Energieversorgung und draht- loser Signalübertragung - Vorträge des 6. GMMWorkshops, Hamburg

[2] U. Marschner, H. Grätz, B. Jettkant, D. Ruwisch, G. Woldt, W.-J. Fischer, B. Clasbrummel, Integration of a wireless lock-in measurement of hip prosthesis vibrations for loosening detection, Sensors and Actuators A: Physical, 156 (2009) 145.

[3] Systemarchitektur intelligenter Sensorimplantate, T. Basmer, P. Kulse, M. Birkholz, Biomedical Engineering / Zeitschrift für Biomedizinische Technik $\mathbf{5 5}$ (2010) 43.

[4] M. Birkholz, K.-E. Ehwald, R. Ehwald, M. Kaynak, J. Borngräber, J. Drews, U. Haak, J. Klatt, E. Matthus, G. Schoof, K. Schulz, B. Tillack, W. Winkler, and D. Wolansky. Mikroviskosimeter zur kontinuierlichen Glucosemessung bei Diabetes mellitus. In: H. Seidel, H. Reichl, and W. Lang (Eds.) Mikrosystemtechnik Kongress 2009, pp. 124, 2009. VDE, VDE Verlag $\mathrm{GmbH}$, Berlin.

[5] MSP430G2x53, MSP430G2x13 Mixed Signal Microcontroller, Texas Instruments, Datasheet, April 2011

[6] ZL70102 Medical Implantable RF Transceiver MICS RF Telemetry, Zarlink Semiconductor, Datasheet, June 2010

[7] LiS 3150M LITRONIK Li-Manganese Dioxide Medium Rate Battery, Datasheet, Litronik, February 2010

*present address: Otto-von-Guericke University, Magdeburg, Germany 